\title{
Aplastic anemia and concomitant autoimmune diseases
}

\author{
Magnus P. Stalder • Alicia Rovó • Jörg Halter • \\ Dominik Heim • Tobias Silzle • Jakob Passweg • \\ Johannes Rischewski • Martin Stern • Caroline Arber • \\ Andreas Buser • Sandrine Meyer-Monard • \\ André Tichelli • Alois Gratwohl
}

Received: 5 September 2008 /Accepted: 11 December 2008/Published online: 13 January 2009

(C) Springer-Verlag 2009

\begin{abstract}
The association of aplastic anemia (AA) with other autoimmune diseases (AID) has been described but so far not systematically evaluated. We assessed the incidence and the outcome of concomitant AID in a retrospective, single-center study of 243 patients with severe AA treated between 1974 and 2006 with either immunosuppression (186) or hematopoietic stem cell transplantation (57) and a median follow-up time of 9.3 years $(0-33)$. Clinically manifest AID were observed in 24 out of $243(10 \pm 3.7 \%)$ patients. Age at diagnosis of AA was significantly younger in patients without AID compared to patients with AID (median, 20 versus 52 years; $P<0.001$ ). In 12 patients where the diagnosis of AID was done before AA therapy, response to antithymocyte globulin was good for AA (ten out of 12) but not for AID (2 out of 12). In 13 patients in which AID occurred after first-line therapy, the median time to the AID was 7 years (range 3 months-27.5 years).
\end{abstract}

Keywords Aplastic anemia - Autoimmune diseases . Antithymocyte globulin

M. P. Stalder · A. Rovó · J. Halter · D. Heim · T. Silzle •

J. Passweg $\cdot$ J. Rischewski $\cdot$ M. Stern $\cdot$ C. Arber $\cdot$ A. Buser $\cdot$

S. Meyer-Monard · A. Tichelli $\cdot$ A. Gratwohl

Basel Stem Cell Transplant Team, University Hospital Basel,

Basel, Switzerland

\section{A. Rovó $(\bowtie)$}

Division of Hematology, University Hospital Basel,

Petersgraben 4,

CH-4031 Basel, Switzerland

e-mail: rovoa@uhbs.ch

\section{Introduction}

Aplastic anemia (AA) is defined as a pancytopenia with unexplained bone marrow hypocellularity. Acquired AA can be considered in most cases as a $\mathrm{T}$ cell-mediated autoimmune disorder, targeted against the hematopoietic progenitors, leading to the failure of the bone marrow [1]. Viral infections, drugs, chemical exposure, pregnancy, or unknown agents seem to trigger the autoimmune dysregulation in patients with predisposition. Associations of AA and other autoimmune diseases (AID) have been shown in single case reports $[2,3]$. However, so far, there are no published data on a systematic review of concomitant AID in AA patients. Moreover, data on the impact of immunosuppressive strategies to treat AA, e.g., antithymocyte globulin (ATG) and cyclosporine A (CSA) on the outcome of AID, are scarce [4].

We sought to determine the incidence and characteristics of concomitant AID diagnosed before or during the course of AA and to compare AA patients with and without AID.

\section{Materials and methods}

This single-center, retrospective cohort study included all 243 patients with the diagnosis of AA, treated at the University Hospital Basel, between 1974 and 2006. The severity of AA was defined according to the widely accepted criteria described by Camitta [5]. The general treatment strategy was uniform with only minor changes throughout the observation period. Patients younger than 40 years with a matched sibling donor received hematopoietic stem cell transplantation (HSCT) as first-line therapy. Older patients and without an eligible sibling donor were treated with intensive immunosuppression 
containing ATG [6] with or without CSA. Since 2001, patients allocated to immunosuppression were included in the prospective European study and randomized to receive ATG and CSA with or without granulocyte colonystimulating factor [7]. In order to circumvent serum sickness of ATG, most patients received steroids during the early phase of the treatment. Patients with relapse or nonresponse (NR) at 3 months usually were splenectomized [8] and, if necessary, retreated with a second course of ATG [9]. Response of AA to therapy was defined according to the Camitta criteria [10]: Splenectomy was performed in nonresponder patients before second-line therapy.

Concomitant AID were defined according to international diagnostic criteria [11-17]. We retained in this study only clinically evident AID. Isolated positive antibody titers without further clinical signs were not included. Response criteria for the concomitant AID were those used for patients with autoimmune disorders treated with HSCT, e.g., absence of all clinical signs without additional therapy and normalization of laboratory values [18]. Patients were controlled yearly; clinical outcome data were collected prospectively and stored in our local database. To identify AID, medical records were systematically reviewed. This study was approved by local Institutional Review Boards.

\section{Statistical analysis}

Left-truncated Cox models were used to assess the impact of patients' characteristics (gender, presence of HLA-DR2, severity of AA, type of AA treatment [ATG, HSCT], splenectomy) on the probability of developing an AID. Severity of AA, AA treatment, and splenectomy were coded as time-dependent covariates. Similarly, the impact of development of AID on survival was assessed by coding AID as a time-dependent risk factor in a Cox model. $P$ values $<0.05$ were considered significant.

\section{Results}

Of the 243 patients, there were 115 (47\%) females. The median age at diagnosis was 20 years (range 1-80 years), the median follow-up time was 9.3 years $(0-33)$. Very severe AA was diagnosed in $128(53 \%)$ patients and severe AA in $115(47 \%)$. Splenectomy was performed in $89(37 \%)$ patients. First-line therapy was ATG in $186(77 \%)$ patients and HSCT in 57 (23\%) (Table 1). In 24 out of $243(10 \%)$ AA patients, a concomitant AID was diagnosed. Thirteen of the patients (54\%) had an AID before diagnosis of AA, and $11(46 \%)$ after therapy for AA. Four out of these 24 patients had more than one AID. In two of them, the first AID was diagnosed before AA appearance and a second AID after first-line therapy. We identified 16 different types of AID (Table 2). The most frequent AID were autoimmune gastritis (six patients) and autoimmune thyroiditis (six patients). The median age at diagnosis of AA was significant lower for patients without AID than patients with a concomitant AID (20 versus 52 years; $P<0.001$ ) (Fig. 1a, b). In our cohort, the cumulative incidence according to the age of the patients at diagnosis of AA increases mainly during the first three decades of age. In

Table 1 Pretreatment patients' characteristics comparing patients with and without concomitant AID

\begin{tabular}{|c|c|c|c|c|}
\hline & All patients & Without AID & With AID & $P$ value \\
\hline Number of patients, $n(\%)$ & 243 & $219(90)$ & $24(10)$ & \\
\hline Median age at diagnosis, years (range) & $20(1-80)$ & $20(1-80)$ & $51.5(9-75)$ & $<0.001^{\mathrm{a}}$ \\
\hline Median follow-up, years (range) & $9.3(0-33)$ & $9.3(0-33)$ & $11.5(0-32)$ & $0.219^{\mathrm{a}}$ \\
\hline \multicolumn{5}{|l|}{ Sex, $n(\%)$} \\
\hline Female & $115(47)$ & $106(46)$ & $9(38)$ & \multirow[t]{2}{*}{$0.310^{\mathrm{b}}$} \\
\hline Male & $128(53)$ & $113(54)$ & $15(62)$ & \\
\hline \multicolumn{5}{|l|}{ Severity, $n(\%)$} \\
\hline Severe AA & $115(47)$ & $101(46)$ & $14(58)$ & \multirow[t]{2}{*}{$0.255^{\mathrm{b}}$} \\
\hline Very severe AA & $128(53)$ & $118(54)$ & $10(42)$ & \\
\hline Splenectomy, $n(\%)$ & $89(37)$ & $81(37)$ & $8(33)$ & $0.723^{\mathrm{b}}$ \\
\hline HLA DRB $1 * 15$ present, $n(\%)$ & $32(49)(n=65)$ & $21(47)(n=45)$ & $11(55)(n=20)$ & $0.598^{\mathrm{b}}$ \\
\hline \multicolumn{5}{|l|}{ First-line treatment, $n(\%)$} \\
\hline ATG & $186(77)$ & $164(75)$ & $22(92)$ & \multirow[t]{2}{*}{$0.019^{\mathrm{b}}$} \\
\hline HSCT & $57(23)$ & $55(25)$ & $2(8)$ & \\
\hline Need for a second-line treatment, $n(\%)$ & $68(28)$ & $63(29)$ & $5(21)$ & $0.441^{\mathrm{b}}$ \\
\hline
\end{tabular}

${ }^{a}$ Mann-Whitney $U$ test

${ }^{\mathrm{b}}$ Chi-square according to Pearson 


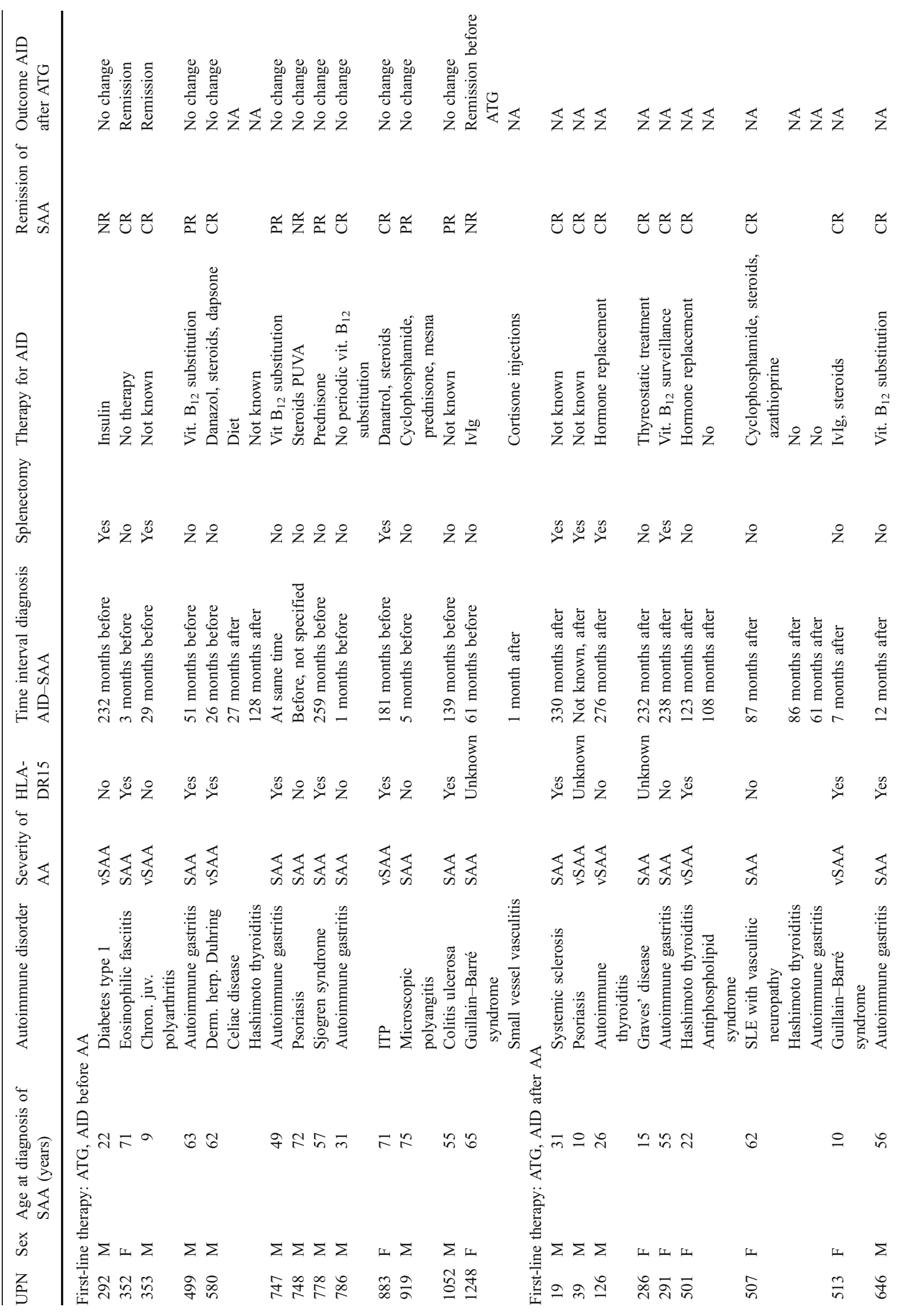




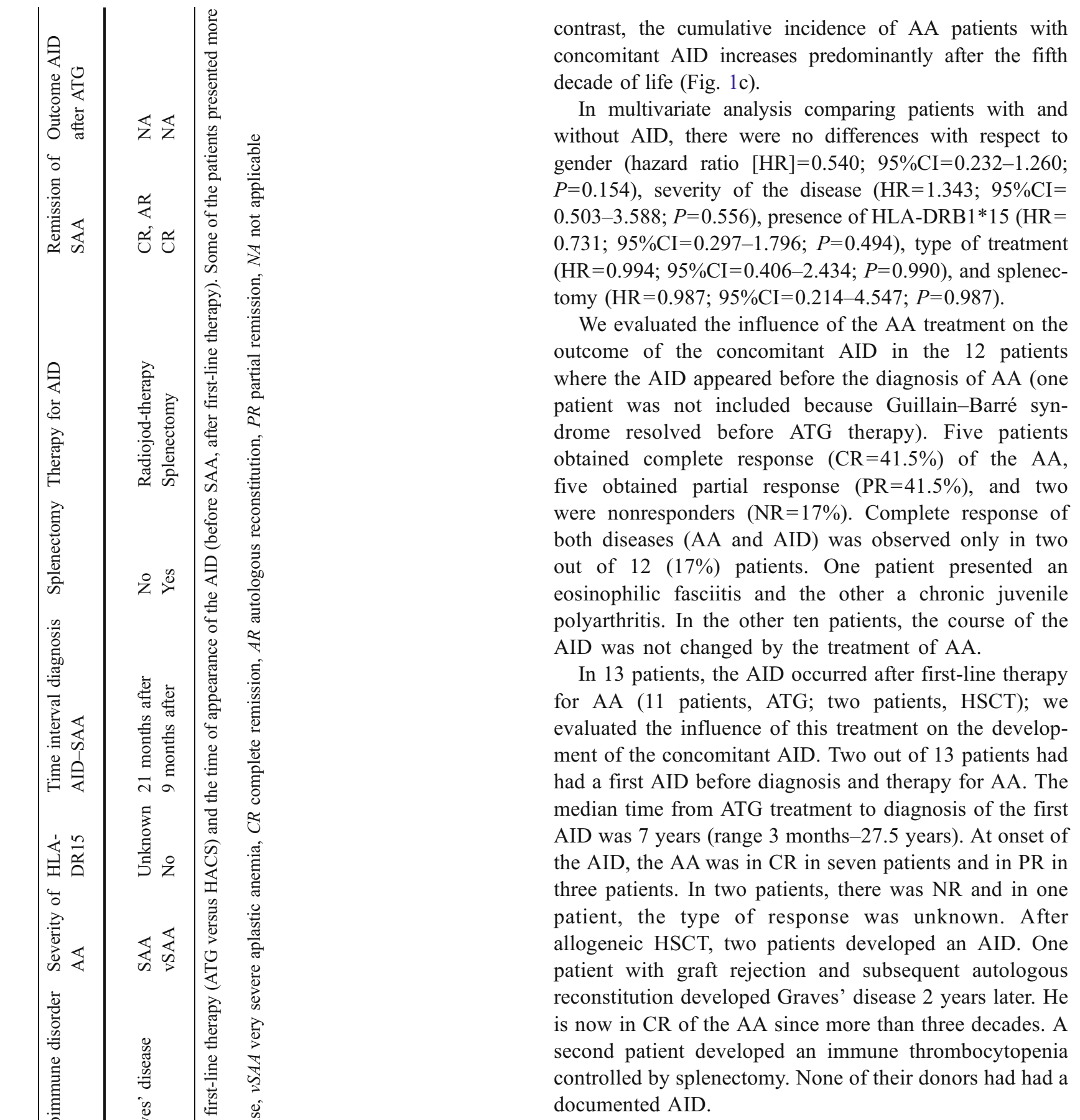

\section{Discussion}

In this study, we show that about one in ten AA patients will develop a concomitant AID during their lifetime, which can appear at any time before and/or after the onset of the AA. The frequency of a concomitant AID is higher in older AA patients. Hence, more than $25 \%$ of AA patients diagnosed after 50 years of age presented a concomitant AID. The main type of concomitant AID appeared to be either gastritis or thyroiditis. AA response to ATG was 
Fig. 1 Age repartition at diagnosis of AA without AID (a) and with AID (b). The data are presented in percentage of the whole group. Patients are divided into three age groups: $<20$, 20-50, and $>50$ years $(P<0.001)$. AA patients without AID are significantly younger than AA patients with a concomitant AID. c Cumulative incidence of AA and the concomitant AID according to the age at diagnosis of each disease. The slope of the curve is different for both diseases. In AA, the cumulative incidence increases mainly during the first three decades of age, whereas in concomitant AID, the cumulative incidence increases predominantly after the fifth decade of life
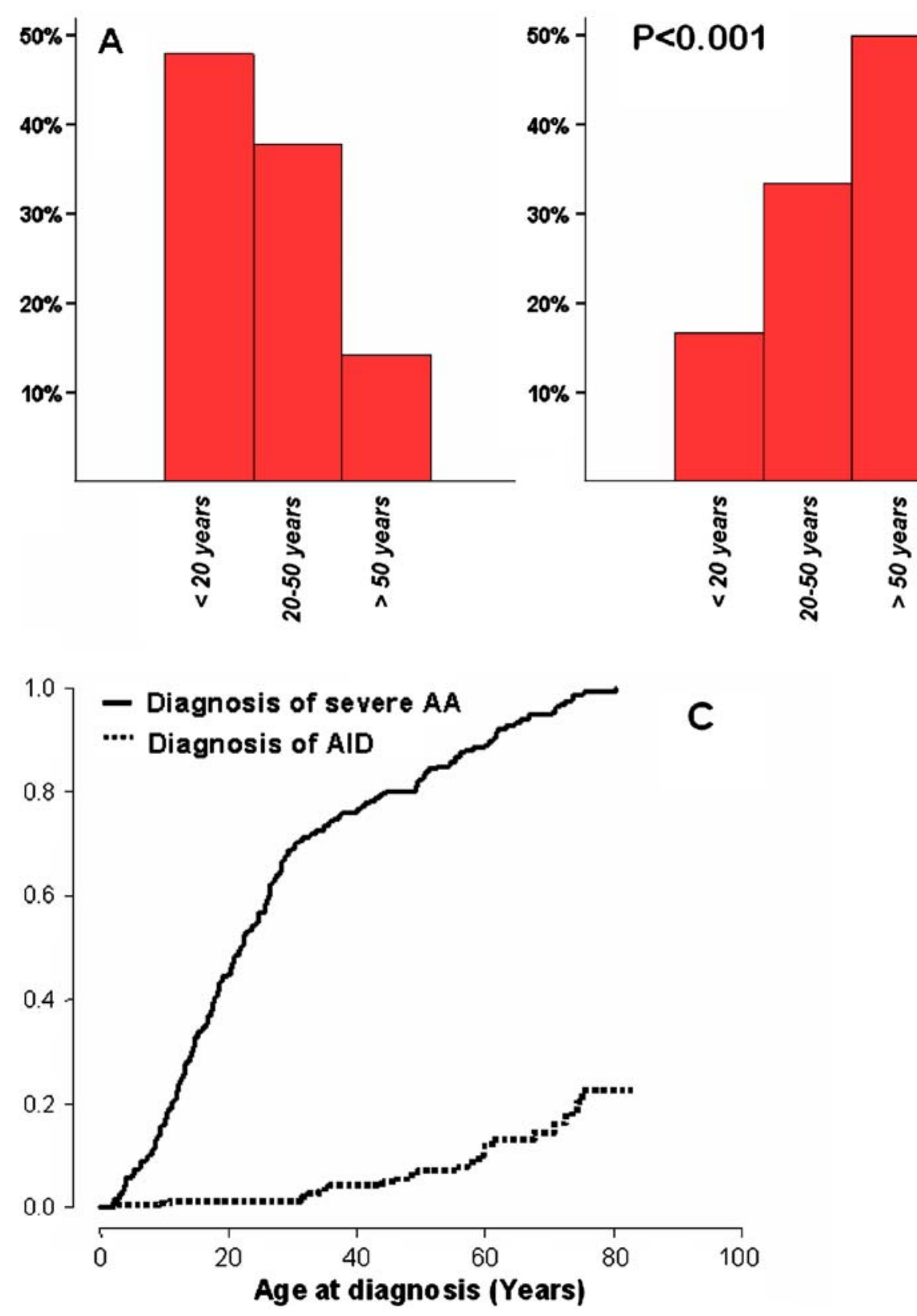

similar in patients with or without AID, but AID response to ATG was poor.

There are some particular features of the AID occurring in AA patients. In contrast to the general population where AID are more common in females [19], in AA patients, AID were more frequently observed in males ( 15 out of 24 , $62 \%$ ). Interestingly, we did not observe cases of rheumatoid arthritis and only one case of systemic lupus erythematosus. HLA-DRB $1 * 15$ has been shown to be involved in the development and the outcome of AA and other autoimmune disorders [20-23]. It seems unlikely that HLADRB $1 * 15$ plays a relevant role in the appearance of concomitant AID in AA patients.
Most cases of acquired AA can be considered as autoimmune disorders characterized by $\mathrm{T}$ cell-mediated, organ-specific destruction of bone marrow hematopoietic cells. However, the usual trigger of this autoimmune reaction remains unclear. In individual patients, the aberrant immune response can sometimes be linked to a viral infection or to drug or chemical exposure. There is much less evidence for other mechanisms including the association with other AID. In consideration of the high frequency of a concomitant AID in AA patients, it is unlikely that both diseases appear together just by chance. It, therefore, raises the question of related pathophysiologic mechanisms. In posthepatitis AA, which typically occurs in young, 
healthy males with self-limited but severe liver inflammation, a common inciting infectious cause could be involved [24]. Indeed, in hepatitis-associated AA, similar skewed T cell repertoires have been detected in the liver and in the peripheral blood lymphocytes, suggesting that a similar antigen-driven pathogenic mechanism is involved for both diseases [25]. This might be different for the concomitant AIDs. Here, there are arguments in favor of distinct mechanisms: the different age repartition of AA patients with and without concomitant AID; the nonresponse of the AID in patients responding to immunosuppressive treatment for the AA and the fact that AA is a T cell-mediated AID, while in many of the concomitant AID, autoantibodies are involved. Common genetic backgrounds, additional immunogenetic, environmental, or hormonal factors may be responsible for the formation of subsets of AID clustering [26]. The AIDs occurring after successful allogeneic HSCT with full donor chimerism do not probably belong to the same category. Late secondary autoimmune-like phenomena have been described after allogeneic HSCT as a possible consequence of skewed immune reconstitution [27].

The older age of our AA patients with concomitant AID suggests that immunosenescence could play a role. Recent studies in healthy octogenarian patients indicate that the immune system, instead of suffering a generalized deterioration, undergoes a remodeling/readjustment of its major functions. Two divergent phenomena may coexist in immunosenescence: a decrease in the capacity of immune response and, simultaneously, autoantibody production [28].

Our study has limitations arising mainly from its retrospective, single-center character and the lack of a control population. There is a relatively small number of patients at risk; however, considering that $\mathrm{AA}$ is a rare disease, this is the first and largest study reporting on the frequency of concomitant AID in AA patients followed up systematically over a long period of time. The advantage of a single-center study is the homogeneity of therapeutical approaches and the consistent follow-up. At last control, $80 \%$ of the long-term survivors had a follow-up of more than 7 years.

In conclusion, in this study, we show that the development of a concomitant AID is frequent, particularly in older AA patients. The AID may appear at any time before or after the AA, and the outcome of the AA is not impaired by the concomitant AID, but the AID does not usually respond to the immunosuppression applied for the AA. The difference in response to ATG therapy between AA and AID suggests independent underlying immune mechanisms. Alternatively, one of these diseases could be the trigger for a second immune dysregulation.
Acknowledgements This work was supported by grants of the Horten Foundation and the Swiss National Research Foundation grant no. 3200 BO-118/76. We thank Katherine Perret and Mariana Gimpelewicz for the English corrections.

Conflicts of interest The authors declare no competing financial interests.

\section{References}

1. Young NS, Calado RT, Scheinberg P (2006) Current concepts in the pathophysiology and treatment of aplastic anemia. Blood 108:2509-2519

2. Antic M, Lautenschlager S, Itin PH (2006) Eosinophilic fasciitis 30 years after-what do we really know? Report of 11 patients and review of the literature. Dermatology 213:93101

3. Hinterberger-Fischer M, Kier P, Forstinger I, Lechner K, Kornek G, Breyer S et al (1994) Coincidence of severe aplastic anaemia with multiple sclerosis or thyroid disorders. Report of 5 cases. Acta Haematol 92:136-139

4. Lytton SD, Denton CP, Nutzenberger AM (2007) Treatment of autoimmune disease with rabbit anti-T lymphocyte globulin: clinical efficacy and potential mechanisms of action. Ann N Y Acad Sci 1110:285-296

5. Camitta BM, Thomas ED, Nathan DG, Santos G, Gordon-Smith EC, Gale RP et al (1976) Severe aplastic anemia: a prospective study of the effect of early marrow transplantation on acute mortality. Blood 48:63-70

6. Viollier R, Tichelli A (2000) Predictive factors for cure after immunosuppressive therapy of aplastic anemia. Acta Haematol 103:55-62

7. Tichelli A, Schrezenmeier H, Socié G, Marsh J, Chapion K, Passweg J A (2002) radomized controlled study in newly diagnosed severe aplastic anemia patients receiving antilymphocyte globulin, cyclosporin A, with or without G-CSF: a study from the EBMT, Severe Aplastic Anemia Working Party. Available at http://www.ebmt.org/5WorkingParties/AAWP/wparties-aa5.html

8. Speck B, Tichelli A, Widmer E, Harder F, Kissling M, Wursch A et al (1996) Splenectomy as an adjuvant measure in the treatment of severe aplastic anaemia. $\mathrm{Br} \mathrm{J}$ Haematol 92:818-824

9. Tichelli A, Passweg J, Nissen C, Bargetzi M, Hoffmann T, Wodnar-Filipowicz A et al (1998) Repeated treatment with horse antilymphocyte globulin for severe aplastic anaemia. $\mathrm{Br} \mathrm{J}$ Haematol 100:393-400

10. Camitta BM (2000) What is the definition of cure for aplastic anemia? Acta Haematol 103:16-18

11. Albers JW, Kelly JJ Jr (1989) Acquired inflammatory demyelinating polyneuropathies: clinical and electrodiagnostic features. Muscle Nerve 12:435-451

12. Boin F, Hummers LK (2008) Scleroderma-like fibrosing disorders. Rheum Dis Clin North Am 34:199-220

13. Dayan CM, Daniels GH (1996) Chronic autoimmune thyroiditis. N Engl J Med 335:99-107

14. Hochberg MC (1995) Classification criteria for childhood arthritic diseases. J Rheumatol 22:1445-1446

15. McMillan R (2007) The pathogenesis of chronic immune thrombocytopenic purpura. Semin Hematol 44:S3-S11

16. Nikolaus S, Schreiber S (2007) Diagnostics of inflammatory bowel disease. Gastroenterology 133:1670-1689 
17. Strickland RG, Mackay IR (1973) A reappraisal of the nature and significance of chronic atrophic gastritis. Am J Dig Dis 18:426-440

18. Gratwohl A, Passweg J, Bocelli-Tyndall C, Fassas A, van Laar JM, Farge D et al (2005) Autologous hematopoietic stem cell transplantation for autoimmune diseases. Bone Marrow Transplant 35:869-879

19. Eaton WW, Rose NR, Kalaydjian A, Pedersen MG, Mortensen PB (2007) Epidemiology of autoimmune diseases in Denmark. J Autoimmun 29:1-9

20. Callander M, Haghighi S, Landtblom AM, Ahlgren CE, Nilsson SI, Rydberg L et al (2007) Multiple sclerosis immunopathic trait and HLA-DR(2)15 as independent risk factors in multiple sclerosis. Mult Scler 13:441-445

21. Graham RR, Ortmann W, Rodine P, Espe K, Langefeld C, Lange E et al (2007) Specific combinations of HLA-DR2 and DR3 class II haplotypes contribute graded risk for disease susceptibility and autoantibodies in human SLE. Eur J Hum Genet 15:823-830

22. Kapustin SI, Popova TI, Lyschov AA, Togo AV, Abdulkadyrov KM, Blinov MN (1997) HLA-DR2 frequency increase in severe aplastic anemia patients is mainly attributed to the prevalence of DR15 subtype. Pathol Oncol Res 3:106-108
23. McCombe PA, Csurhes PA, Greer JM (2006) Studies of HLA associations in male and female patients with Guillain-Barre syndrome (GBS) and chronic inflammatory demyelinating polyradiculoneuropathy (CIDP). J Neuroimmunol 180:172-177

24. Brown KE, Tisdale J, Barrett AJ, Dunbar CE, Young NS (1997) Hepatitis-associated aplastic anemia. N Engl J Med 336:10591064

25. Lu J, Basu A, Melenhorst JJ, Young NS, Brown KE (2004) Analysis of T-cell repertoire in hepatitis-associated aplastic anemia. Blood 103:4588-4593

26. Theander E, Jacobsson LT (2008) Relationship of Sjogren's syndrome to other connective tissue and autoimmune disorders. Rheum Dis Clin North Am 34:935-947

27. Trendelenburg M, Gregor M, Passweg J, Tichelli A, Tyndall A, Gratwohl A (2001) Altered immunity syndrome, a distinct entity in long-term bone marrow transplantation survivors? Bone Marrow Transplant 28:1175-1176

28. Ramos-Casals M, Garcia-Carrasco M, Brito MP, Lopez-Soto A, Font J (2003) Autoimmunity and geriatrics: clinical significance of autoimmune manifestations in the elderly. Lupus 12:341-355 